\title{
RESEARCH
}

Open Access

\section{Dynamic perfusion digital radiography for predicting pulmonary function after lung cancer resection}

\author{
Jun Hanaoka ${ }^{1 *}$, Makoto Yoden ${ }^{1}$, Kazuki Hayashi', Takuya Shiratori ${ }^{1}$, Keigo Okamoto ${ }^{1}$, Ryosuke Kaku', \\ Yo Kawaguchi', Yasuhiko Ohshio ${ }^{1}$ and Akinaga Sonoda ${ }^{2}$
}

\begin{abstract}
Background: Accurate prediction of postoperative pulmonary function is important for ensuring the safety of patients undergoing radical resection for lung cancer. Dynamic perfusion digital radiography is an excellent and easy imaging method for detecting blood flow in the lung compared with the less-convenient conventional lung perfusion scintigraphy. As such, the present study aimed to confirm whether dynamic perfusion digital radiography can be evaluated in comparison with pulmonary perfusion scintigraphy in predicting early postoperative pulmonary function and complications.
\end{abstract}

Methods: Dynamic perfusion digital radiography and spirometry were performed before and 1 and 3 months after radical resection for lung cancer. Correlation coefficients between blood flow ratios calculated using dynamic perfusion digital radiography and pulmonary perfusion scintigraphy were then confirmed in the same cases. In all patients who underwent dynamic perfusion digital radiography, the correlation predicted values calculated from the blood flow ratio, and measured values were examined. Furthermore, ppo\%FEV1 or ppo\%DLco values, which indicated the risk for perioperative complications, were examined.

Results: A total of 52 participants who satisfied the inclusion criteria were analyzed. Blood flow ratios measured using pulmonary perfusion scintigraphy and dynamic perfusion digital radiography showed excellent correlation and acceptable predictive accuracy. Correlation coefficients between predicted FEV1 values obtained from dynamic perfusion digital radiography or pulmonary perfusion scintigraphy and actual measured values were similar. All patients who underwent dynamic perfusion digital radiography showed excellent correlation between predicted values and those measured using spirometry. A significant difference in ppo\%DLco was observed for respiratory complications but not cardiovascular complications.

Conclusions: Our study demonstrated that dynamic perfusion digital radiography can be a suitable alternative to pulmonary perfusion scintigraphy given its ability for predicting postoperative values and the risk for postoperative respiratory complications. Furthermore, it seemed to be an excellent modality because of its advantages, such as simplicity, low cost, and ease in obtaining in-depth respiratory functional information.

(Continued on next page)

\footnotetext{
* Correspondence: hanaoka@belle.shiga-med.ac.jp

'Division of General Thoracic Surgery, Department of Surgery, Shiga

University of Medical Science, Shiga, Japan

Full list of author information is available at the end of the article
}

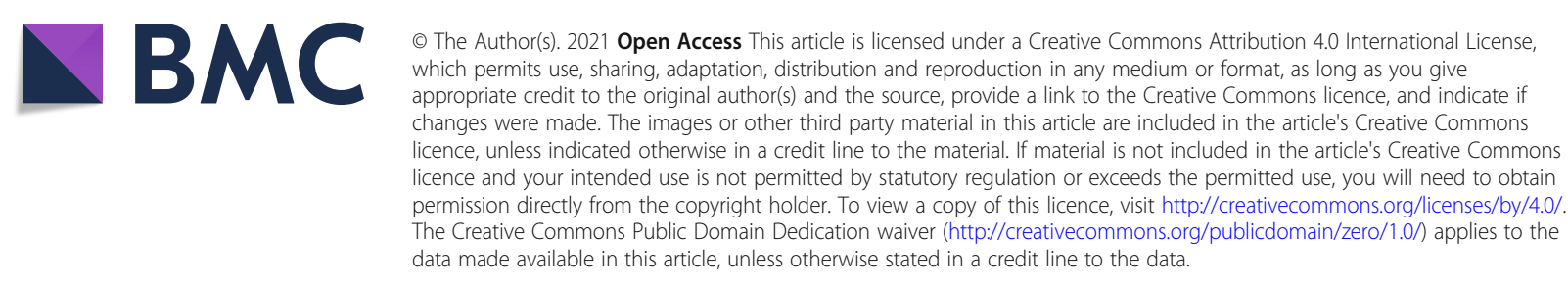




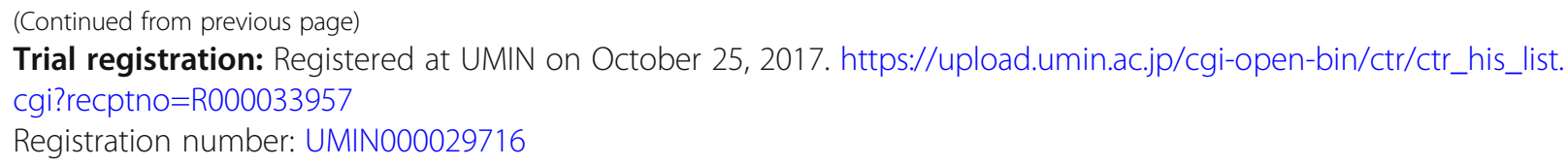

Keywords: Dynamic perfusion digital radiography, Dynamic chest radiography, Pulmonary perfusion scintigraphy, Pulmonary perfusion, Prediction of postoperative respiratory function

\section{Background}

Lung cancer, one of the most commonly diagnosed cancers, remains the leading cause of cancer-related death across the world [1]. Although a number of therapeutic options for lung cancer, such as surgery, radiotherapy, and chemotherapy, are available, pulmonary lobectomy with lymph node dissection is necessary for the treatment of primary non-small-cell lung cancer. Considering that respiratory impairment after lung resection is unavoidable, preoperative assessment of cardiovascular and lung functions is important for ensuring perioperative safety and maintenance of the activities of daily living [1-3]. Especially considering the increasing number of elderly patients or those with coexisting obstructive lung disease or interstitial lung disease related to smoking, it is important to ensure safety throughout the surgical procedure and maintenance of the daily living activities [1]. Predicting postoperative lung function involves predicting the actual values $[4,5]$ and evaluating the risk factors for mortality and morbidity after the surgery $[2$, 3]. Spirometry, diffusing capacity for carbon monoxide (DLco), and maximal oxygen consumption are important for determining a patient's preoperative respiratory function. In clinical practice, a predicted postoperative percent forced expiratory volume in $1 \mathrm{~s}$ (ppo\%FEV1) calculated using a method based on lung segment counting and pulmonary perfusion scintigraphy (PPS) by quantifying the pulmonary blood flow distribution to improve the predictive accuracy has shown good correlation with the actual measured postoperative values [410]. Furthermore, ppo\%FEV1 and ppo\%DLco have shown a strong correlation with long-term postoperative prognosis [2] and have been used in risk assessment algorithms created using exercise stress tests as an index [3]. Although angiography, ultrasonography, quantitative computed tomography, and dynamic magnetic resonance imaging have been used to visualize or quantify the blood flow in the lungs [11-13], PPS has been considered as the most reliable and conventional method for measuring the pulmonary blood distribution. The American College of Chest Physicians guidelines recommend assessing the risk for surgery using a functional algorithm, including ppo\%FEV1 and ppo\%DLco values as calculated using quantitative radionuclide perfusion scanning [14]. Even the conventionally used PPS, which is highly reliable, poses various drawbacks, such as requiring significant investment in equipment, exposure dose to radioisotopes, and ingenuity in the shooting method. Dynamic chest radiography (DCR) using dynamic flat-panel detectors with a large field of view and advanced digital image processing can provide sequential chest radiographs with high temporal resolution during the respiratory cycle and allows the quantification of pulmonary blood flow distribution from the amount of pixel value changes in the lung field associated with the cardiac cycle [15]. Furthermore, computerized DCR methods, unlike other modality, can provide information regarding pulmonary ventilation and circulation by measuring the slight changes in the pixel value, without the use of contrast media or radioisotope [16]. We specifically named this method as dynamic evaluation of pulmonary circulation for "Dynamic perfusion digital radiography (DPDR)". DPDR is considered to be a very useful examination method because it can simultaneously provide not only qualitative and quantitative information on dynamic pulmonary circulation but also information on pulmonary ventilation or movement of the diaphragm and chest wall despite its low cost, short examination time, low exposure, and simple imaging method [17]. This circulation image obtained from DCR revealed a normal pattern, which diffuses from the pulmonary arteries to the peripheral area. Nonetheless, whether this method can be used to quantify the pulmonary blood flow needs to be determined by comparing it with the conventional method for evaluating PPS.

The present study aimed to prospectively evaluate whether blood flow imaging on chest DPDR can be a viable substitute for conventional PPS in predicting postoperative pulmonary function. Accordingly, we compared pulmonary blood flow ratios (BFRs) determined through DPDR and conventional PPS and evaluated the correlation between predicted postoperative respiratory function values using DPDR and measured values.

\section{Methods}

\section{Patients}

This prospective study was approved by the Institutional Review Board of Shiga University of Medical Science (CRB 5180008; 10 October 2017). The study was then registered as a clinical trial (UMIN000029716). All 
patients provided written consent after having been informed regarding the research protocol. Patients scheduled for radical resection due to primary lung cancer from May 2018 to November 2019 were recruited. Only those who were able to follow the breathing instructions (which involved breath holding or forced breathing) in a standing or sitting position were included. A total of 57 patients underwent follow-up evaluation 3 months after surgery (February 2020). The exclusion criteria were as follows: (i) patients with a history of thoracic surgery, (ii) those younger than 20 years old, or (iii) those who may develop adverse events due to irradiation. Five patients were excluded due the following reasons: presence of disease other than lung cancer $(n=2)$, wedge resection of the lung $(n=1)$, lost to follow-up $(n=1)$, and refused informed consent before the postoperative period $(n=$ 1). A total of 52 participants (40 men, 12 women; mean age $71.7 \pm 7.6$ years; age range $53-83$ years) were ultimately analyzed (Fig. 1). Among these, 27 underwent PPS before surgery. Patient data collected included age, sex, affected side, lung lobe resected, and complications related to cardiovascular and respiratory systems.

\section{Methods}

\section{Imaging protocol for dynamic chest radiology}

Posteroanterior DCR was performed using a prototype system (Konica Minolta, Inc., Tokyo, Japan) composed of an indirect-conversion flat-panel detector (PaxScan, 4343CB, Varex Imaging Corporation, Salt Lake City, UT, USA), an X-ray tube (RAD-94/B-130H, Varian Medical Systems, Inc., Palo Alto, CA, USA), and a pulsed X-ray generator (EPS45RF, EMD Technologies, SaintEustache, Canada). All participants were scanned in a sitting position for approximately $10 \mathrm{~s}$ while holding their breath. The exposure conditions were as follows: tube voltage, $100 \mathrm{kV}$; tube current, $40 \mathrm{~mA}$; duration of pulsed X-ray, $5 \mathrm{~ms}$; source-to-image distance, $2 \mathrm{~m}$; additional filter, $0.5 \mathrm{~mm} \mathrm{Al}+0.1 \mathrm{~mm} \mathrm{Cu}$. Matrix size was $1024 \times 1024$ pixels, pixel size was $417 \times 417 \mu \mathrm{m}$, and the whole image area was $42.7 \times 42.7 \mathrm{~cm}$. The pixel value range in each flat-panel detector pixel was 65,536 (16 bit). However, the pixel value was saturated at approximately 58,000 , which corresponds to an entrance surface dose of approximately $1.5 \mu \mathrm{Gy}$. A high frame rate (15 frames/s) was used for capturing perfusioninduced changes in pixel value. The pulsed X-ray protected subjects from excessive radiation exposure. Total radiation exposure was set to be $<1.5 \mathrm{mGy}$, which is the International Atomic Energy Agency guidance level for both posterior anterior and lateral chest radiographies.

\section{Pulmonary perfusion analysis}

The similarity between pixel value changes in the ventricle (heartbeat waveform) and those in the lungs was

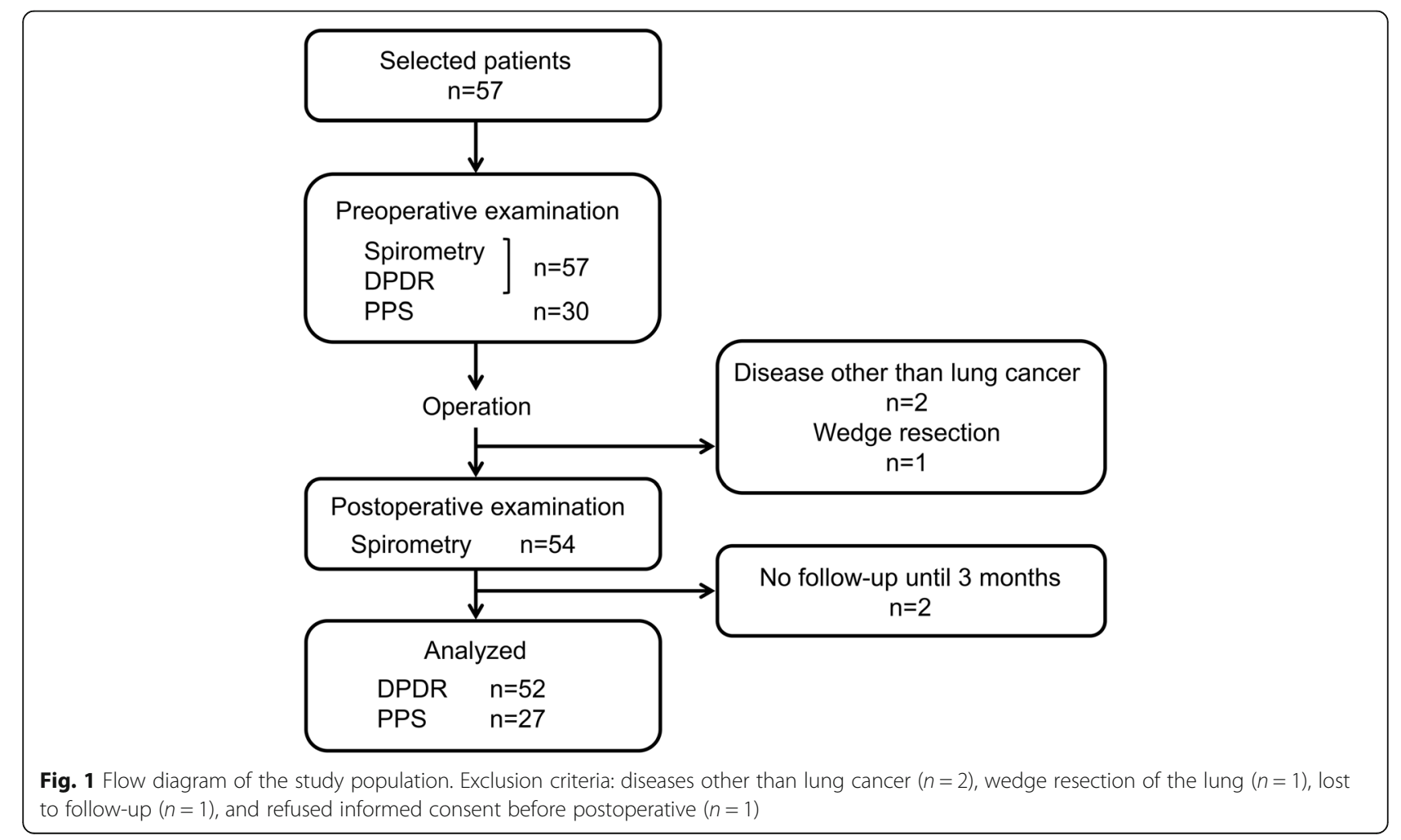


determined using the following cross-correlation method. First, pixel value changes corresponding to a respiratory cycle were removed using a high-pass filter (cutoff frequency $0.85 \mathrm{~Hz}$ ) to extract a periodic pixel value change corresponding to a cardiac cycle. Second, a $25 \times 25 \mathrm{~mm}$ region of interest (ROI) was established on the ventricle region, after which the temporal change in pixel value was measured ( $\mathrm{PC}_{-}$ $\operatorname{lv}(t))$. Last, the temporal change in pixel value was measured on each pixel at $(x, y)$ in the lung field ( $\mathrm{PC}_{-}$ lf $(x, y, t))$. The cross-correlation value (CCv) between $-1.0 \times$ PC_lv and PC_lf for all pixels in the lung field was calculated frame by frame (PC_lv should be an inverse of PC_lf). The obtained $\mathrm{CCv}$ (from -1.0 to 1.0) at each frame was color-coded. The presence of a waveform shape similar to PC_lv indicates the presence of blood flow. A lower correlation indicates less blood flow, while a higher correlation indicates more blood flow (Fig. 2).

\section{Blood flow ratio measurement}

The maximum $\mathrm{CCv}$ (MaxCCv) of each pixel was calculated for all frames, after which the sum of $\mathrm{MaxCCv}$ for both left and right lung fields were calculated (SumMaxCCv_left, SumMaxCCv_right). BFR was defined as follows when the left lung was affected (Eq. 1) and when the right lung was affected (Eq. 2):
BFR $=$ SumMaxCCv_left/(SumMaxCCv_right + SumMaxCCv_left $)$

BFR = SumMaxCCv_right $/($ SumMaxCCv_right + SumMaxCCv_left $)$

\section{Pulmonary perfusion scintigraphy}

A dual-head, variable-angle gamma camera (Discovery630; GE Healthcare Life Sciences, Amersham Place, Little Chalfont, Buckinghamshire HP7 9NA, England) with high-resolution low-energy collimators acquired with a $256 \times 256$ matrix size (zoom 1.0) was used to perform lung perfusion scintigraphy. To ensure optimal radiopharmaceutical distribution in the lungs, each patient initially received half of the $200-\mathrm{MBq} 99 \mathrm{mTc}$ microalbu$\mathrm{min} / 99 \mathrm{mTc}$-macroaggregate solution in prone position, followed by the other half with the patient in supine position. After intravenous administration, planar scans were obtained in eight projections: anterior, posterior, left lateral, right lateral, right anterior oblique, right posterior oblique, left anterior oblique, and left posterior oblique ( 1 million counts each). Images were processed using an automated software for quantitative perfusion analysis which was available at a Xeleris imageprocessing station. This software automatically divides both lungs into three regions of interest in AP and PA

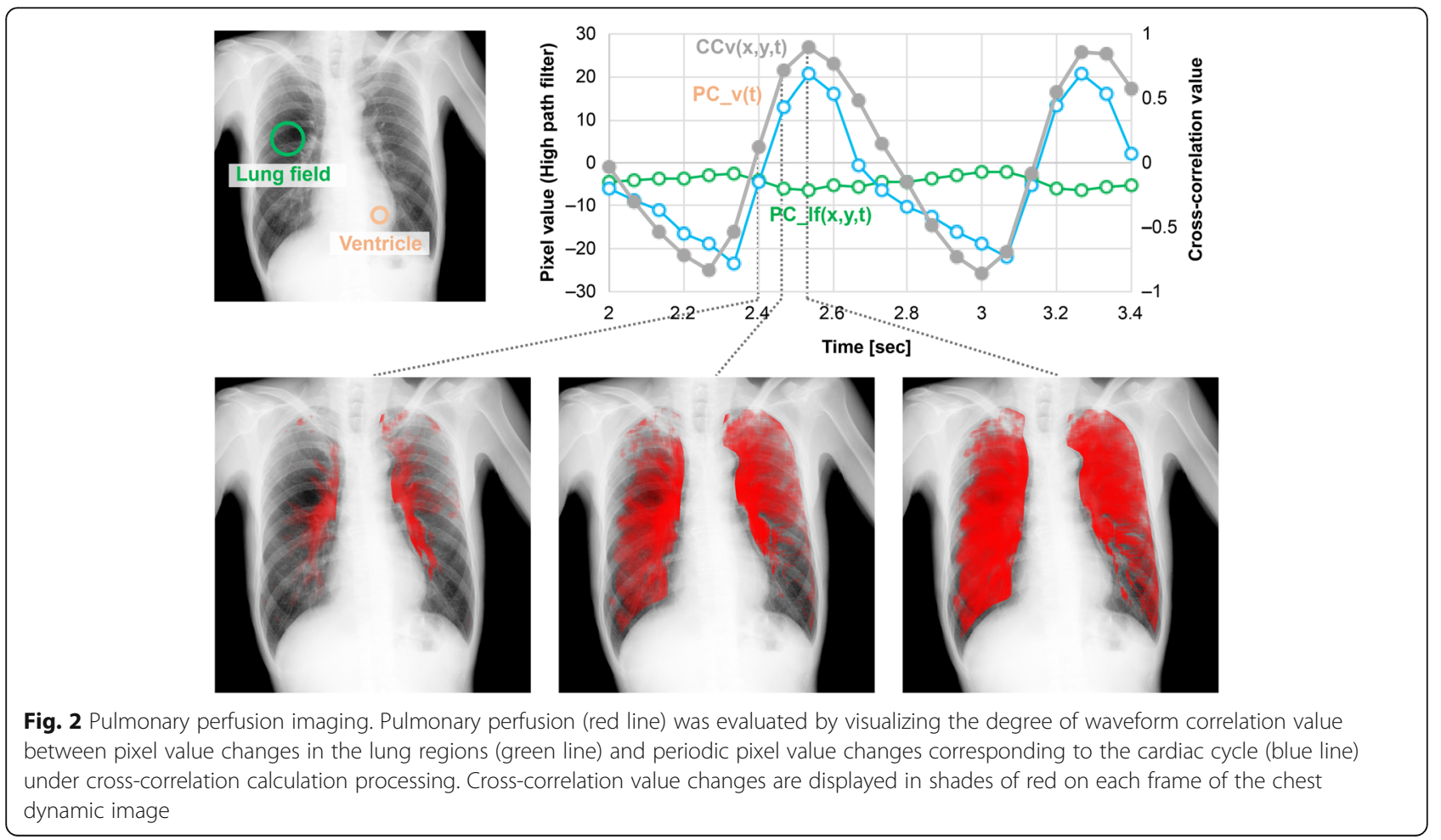


projections and calculates geometric mean values from both projections in all six ROIs.

\section{Pulmonary function tests}

All participants underwent pulmonary function tests within 2 months prior to surgery and at 1 and 3 months after surgery using a computerized spirometer (FUDAC77; Fukuda Denshi Co., LTD, Tokyo, Japan). Pulmonary function test parameters included FEV1, \%FEV1, DLco, and \%DLco values. From the perspective of the frequency of postoperative complications, we selected 1 month after the surgery as an index of surgical safety and 3 months after the surgery as reflecting a high frequency of complications $[18,19]$. At 3 months after surgery, the effects of surgery in several cases seemed withdrawn with the recovery of the postoperative lung functions.

\section{Predicted postoperative lung function using the segment- counting method}

Predicted postoperative lung function values were calculated using a segment-counting formula developed by Ali et al. [20] and Gass GD et al. [21] (Eq. 3):

$$
\begin{aligned}
\text { ppoFEV1 }= & \text { preFEV1 } \\
& \times \frac{\text { Remaining lung segment numbers }}{\text { Total lung segment numbers }}
\end{aligned}
$$

In the segment-counting method, "a" represents the total number of unobstructed segments in the resection lobe, which is assumed to be 3,2 , and 5 for the right upper, middle, and lower lobes, respectively, and 3, 2, and 4 for the left upper segments, lingular segment, and lower lobe, respectively. Overall, 19 segments were used to represent the entire lung. Furthermore, predicted postoperative lung function values were calculated using the left-right BFR from DPDR and PPS through the following formula (Eq. 4):

$$
\begin{aligned}
\text { ppoFEV1 }(\text { DPDR or PPS }) & \\
= & \text { preFEV } 1 \times(\text { BFR of intact side } \\
& +\frac{\text { Remaining lung segment number }}{\text { Total lung segment number of affected side }} \\
& \times \text { BFR of affected side })
\end{aligned}
$$

Correlations between each predicted postoperative value and actual spirometry value were examined at 1 and 3 months after the surgery, with ppoDLco (DPDR or PPS) being calculated at the same time points.

\section{Statistical analysis}

All statistical analyses were performed using SPSS version 25 (Chicago, IL, USA). Data were reported as means \pm standard deviations (SDs). Correlations between BFRs calculated through DPDR and PPS before surgery were evaluated using Pearson's correlation and regression analysis. Furthermore, one version of the Bland-Altman analysis [22] was used to compare BFRs calculated through DPDR and PPS (with certain systematic and random errors). Intervals of agreement were drawn as the mean difference between predicted and measured values $\pm 2 \mathrm{SD}$ of the differences. Correlations between postoperatively measured values and those predicted using the BFR from DPDR and PPS at postoperative months 1 and 3 were examined. Furthermore, the correlation between values predicted using DCR and those measured at postoperative months 1 and 3 were analyzed. Differences between ppo\%FEV1 and ppo\%DLco, which indicate risk for perioperative respiratory complications, were assessed using the unpaired $t$ test. All statistical analyses were two-tailed, with significance being set at 0.05 .

\section{Results}

\section{Clinical characteristics}

A total of 52 patients were analyzed throughout the study period (Fig. 1). Of the total, 50 underwent lobectomy, and two underwent segmentectomy (upper division, S3, and S6). Their clinical characteristics are summarized in Table 1. A total of 30 patients underwent

Table 1 Patients' clinical characteristics

\begin{tabular}{lllll}
\hline Variable & \multicolumn{4}{l}{ No. of patients } \\
\cline { 2 - 5 } & With PPS & $\mathbf{( \% )}$ & Total & (\%) \\
\hline Cases & 27 & $(100.0)$ & 52 & $(100.0)$ \\
Age (years), mean \pm SD & $73.0 \pm 6.8$ & & $71.7 \pm 7.6$ & \\
Gender & & & & \\
$\quad$ Male & 24 & $(88.9)$ & 40 & $(76.9)$ \\
Female & 3 & $(11.1)$ & 12 & $(23.1)$ \\
Affected side & & & & \\
Right & 12 & $(44.4)$ & 30 & $(57.7)$ \\
$\quad$ Left & 15 & $(55.6)$ & 22 & $(42.3)$ \\
Resection & & & & \\
RU & 6 & $(22.2)$ & 18 & $(34.6)$ \\
RM & 2 & $(7.4)$ & 4 & $(7.7)$ \\
$\quad$ RL & 4 & $(14.8)$ & 8 & $(15.4)$ \\
LU & 10 & $(37.0)$ & 16 & $(30.8)$ \\
$\quad$ LL & 5 & $(18.5)$ & 6 & $(11.5)$ \\
Complications & 10 & $(37.0)$ & 19 & $(36.5)$ \\
Respiratory & 10 & $(37.0)$ & 14 & $(26.9)$ \\
Cardiovascular & 1 & $(3.7)$ & 6 & $(11.5)$ \\
\hline
\end{tabular}

PPS pulmonary perfusion scintigraphy, $R U$ right upper lobe, $R M$ right middle lobe, $R L$ right lower lobe, $L U$ left upper lobe, $L L$ left lower lobe 
PPS before surgery, among whom 27 were suitable for final analysis. The resected lobes included the right upper $(n=18)$, right middle $(n=4)$, right lower $(n=8)$, left upper $(n=16)$, and left lower $(n=6)$ lobes. Complications requiring treatment up to 3 months after surgery occurred in 19 cases, among which 14 were respiratory system-related (bacterial pneumonia, interstitial pneumonia, pleuritis, atelectasis, hypoxemia, and prolonged air leakage) and six were cardiovascular system-related (paroxysmal atrial fibrillation). During this observation period, there was no recurrence in the eligible cases, and neither death due to other disease nor death due to cancer occurred.

\section{Blood flow distribution}

The correlation between affected side BFRs obtained from DPDR and PPS was examined in 30 patients before surgery. Figure 3a shows the linear regression analysis of the affected side-to-total ratio obtained using PPS and DPDR. Accordingly, the PPS distribution ratio $(y)$ and DPDR ratio $(x)$ showed excellent correlation $(r=0.829$; $P<0.01)$. Bland-Altman analysis revealed a $1.02 \%$ difference in the means of $93.3 \%$ of the values within 2 SDs of the mean and a proportional error indicating a regression line of $y=-0.238 x+12.535$ occurring due to variations in the smaller average side of the scatter diagram (Fig. 3b). This was attributed to substantial variation in BFRs between DPDR and PPS on the left affected side, which was caused by the effects of a beating heart during the analysis. Based on the above, the BFRs obtained from DPDR and PPS showed a high correlation and were within an acceptable error range, suggesting that DPDR was comparable to PPS in blood flow evaluation.

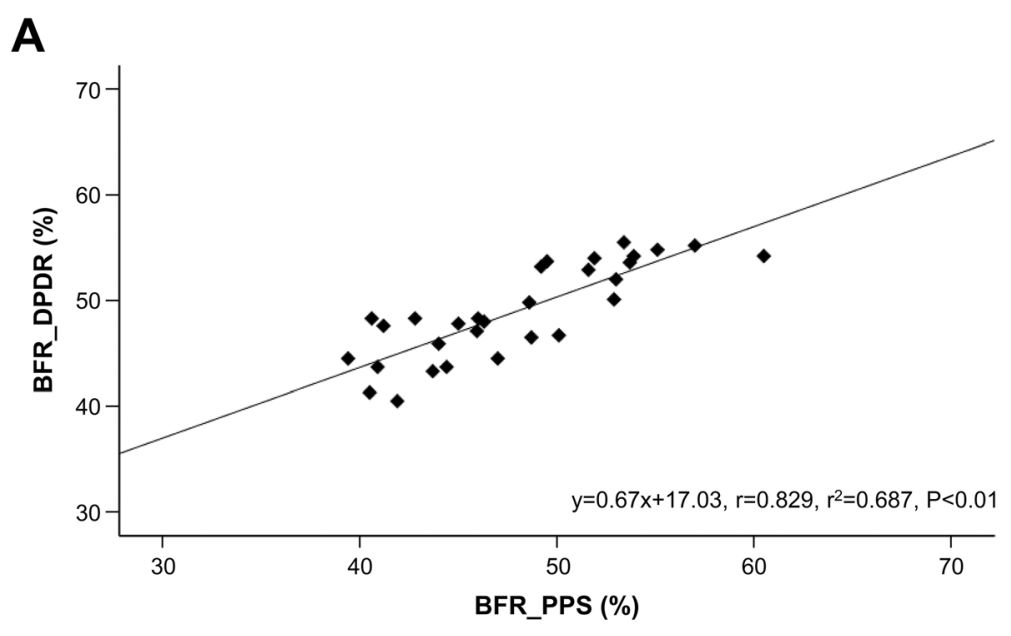

B

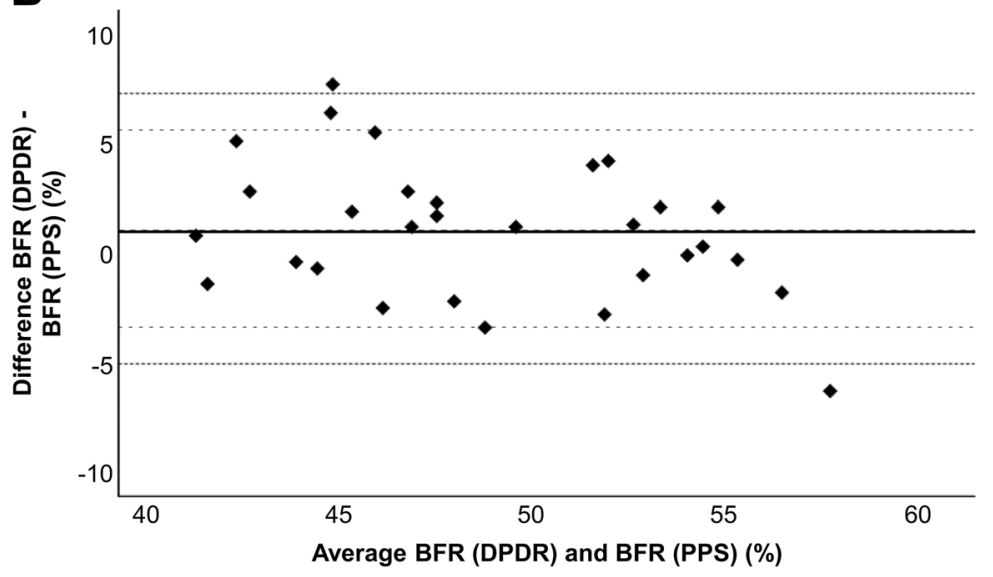

Fig. 3 Comparison between blood flow ratio (BFR) from dynamic perfusion digital radiography and pulmonary perfusion scintigraphy. a Correlation between the BFR on the affected side obtained from dynamic perfusion digital radiography (DPDR) and pulmonary perfusion scintigraphy (PPS). b Bland-Altman analysis between affected side BFR obtained from DPDR and PPS. The black line indicates the mean, the dotted lines indicate the limit of agreement, and the broken lines indicate mean $\pm 2 \mathrm{SD}$ 
Relationship between values measured using spirometry and those predicted using DPDR or PPS

The relationship between the actual measured values and those predicted using BFRs obtained from DPDR and PPS was examined among 27 patients who underwent simultaneous DPDR and PPS. The correlation between FEV1 and DLco values measured using spirometry, which have been frequently used to determine postoperative functional lung capacity and predicted values was examined at postoperative months 1 and 3. Accordingly, the correlation coefficients between predicted values obtained from DPDR or PPS and actual measured values at postoperative months 1 and 3 were similar for FEV1 $(0.885$ and 0.915 on DPDR; 0.895 and 0.915 on PPS, respectively) (Additional file 1). Table 2 presents a summary of the data obtained using DPDR and PPS. Our results also showed high correlation between DPDR and PPS for DLco, with higher correlation coefficients for FEV1 and DLco at postoperative months 3 and 1, respectively. The evaluation of perioperative respiratory status was useful in the early stage of DLco and in the stable period of FEV1. Based on these results, the correlation between values predicted using DPDR in all 52 patients and those measured using spirometry at postoperative months 1 and 3 were examined. Linear regression analysis of actual measured values at postoperative months 1 and 3 for FEV1 and DLco showed excellent correlation $\left(r^{2}=0.816, y=0.945 x+\right.$ 25.836; $r^{2}=0.883, y=1.034 x+(-2.669) ; r^{2}=0.862$, $y=0.942 x+0.502$; and $r^{2}=0.845, y=0.989 x+$ 0.453) (Fig. 4). Our results showed that values predicted using BFR calculated from DPDR was highly correlated with those predicted using PPS and those actually measured during the early postoperative period. Based on the above, the postoperative pulmonary function predicted from DPDR and PPS respectively showed a high correlation with the actual measured value, and there was no difference. Moreover, especially the correlation of the predicted value using DPDR did not decrease even when the number of samples increased, suggesting that DPDR was comparable to PPS in postoperative lung function prediction.

Table 2 Summary of the correlation coefficients between predicted values and actual measured values after surgery

\begin{tabular}{llllll}
\hline & FEV1 & & & DLco & \\
\cline { 2 - 3 } \cline { 5 - 6 } & POM 1 & POM 3 & & POM 1 & POM 3 \\
\hline DPDR & 0.885 & 0.951 & & 0.929 & 0.915 \\
PPS & 0.895 & 0.951 & & 0.930 & 0.917 \\
\hline
\end{tabular}

$D P D R$ dynamic perfusion digital radiography, PPS pulmonary perfusion scintigraphy, FEV1 forced expiratory volume in $1 \mathrm{~s}, D L c o$ diffusing capacity for carbon monoxide, $P O M$ postoperative month
Predicting postoperative complications through predictive lung function

A total of 19 patients (36.5\%) developed postoperative complications requiring treatment, among whom 14 $(26.9 \%)$ were respiratory-related and $6(11.5 \%)$ were cardiovascular-related (Table 1). The mean difference between ppo\%FEV1 and ppo\%DLco values obtained from DPDR, which were calculated using the prediction formula including age and height, was determined using an unpaired $t$ test. Although no significant difference in cardiovascular complications was observed, a significant difference in ppo\%DLco for respiratory complications was noted (Fig. 5). Complication rates for ppo\%FEV1 at $40-70 \%$ and $\geq 70 \%$ were $44.4 \%$ and $17.6 \%$, respectively, excluding values $<40 \%$. Gradual complication rates for ppo\%DLco at $<40 \%, 40-60 \%$, and $\geq 60 \%$ were 50,25 , and $18.2 \%$, respectively. Our results revealed that ppo\%DLco reflected the risk for respiratory complications within 3 months.

\section{Discussion}

The purpose of this study was to evaluate whether DPDR, a new technology, can be applied as a substitute for postoperative pulmonary function prediction and postoperative complication prediction using the conventional PSS.

Our results revealed a strong correlation between PPS and DPDR in the regression analysis of affected side BFR among patients with lung cancer before surgery (Fig. 3a). Furthermore, Bland-Altman analysis revealed that the limit of agreement on optimistic interpretation was between -3.386 and 5.819, indicating an acceptable degree of agreement (Fig. 3b). Moreover, the present study found that values predicted using BFR from DPDR strongly correlated with not only those predicted from PPS but also those actually measured among patients who underwent DPDR during the early postoperative period (Table 2; Fig. 4, Additional file 1). Previous studies had reported that the correlation coefficient for PPS was approximately $0.8-0.9[5,8-10,23,24]$, which is comparable to the results presented herein. Furthermore, our results revealed that ppo\%DLco reflected the risk of respiratory complications within 3 months (Fig. 5). Although some reports show that FEV1 can be a good predictor of postoperative respiratory function, the present study only showed a trend given our exclusion of patients with a ppo\%FEV1 of $<40 \%[2,3,14]$. The current study showed that pulmonary blood flow measurements obtained using DPDR strongly correlated with the results of blood flow scintigraphy, suggesting that DPDR can be a possible substitute for PPS in blood flow distribution analysis.

PPS has been an established method for assessing pulmonary blood flow; however, it may impose a burden on 

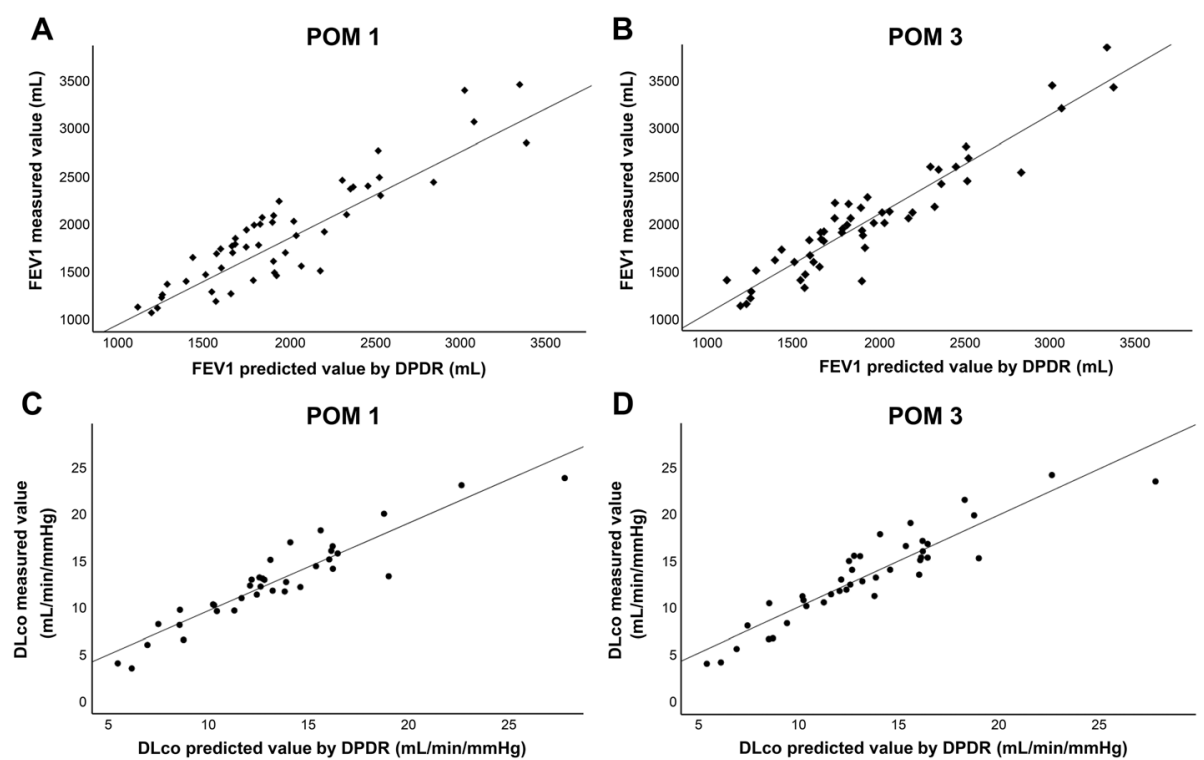

Fig. 4 Comparison between postoperative predicted and measured values of FEV1 and DLco. Correlations between actual postoperatively measured values and those predicted using dynamic perfusion digital radiography (DPDR) for the forced expiratory volume in $1 \mathrm{~s}$ (FEV1) and diffusing capacity for carbon monoxide (DLCo) at postoperative months 1 (a, c) and 3 (b, d)

medical institutions and patients (e.g., facility investment, nuclide preparation, exposure dose, and shooting time). DCR provides visual information on respiratory kinetics and functional imaging of the pulmonary circulation and ventilation [15]. Furthermore, DPDR can be potentially advantageous considering its low dose, small space requirement, and cost effectiveness. Provided that no significant differences in the obtained results are noted, DPDR may be considered to be more convenient than PPS in a clinical setting (see Table 3 for a comparison between DPDR and PPS). The evaluation and imaging methods (procedure) have not yet been established because of a possibility that the physiological functions that can be evaluated will eventually expand.

Until date, DCR has been reported for image evaluation of chronic thromboembolic pulmonary hypertension, for the prediction of tumor from visceral pleura origin, correlation between changes in lung field area
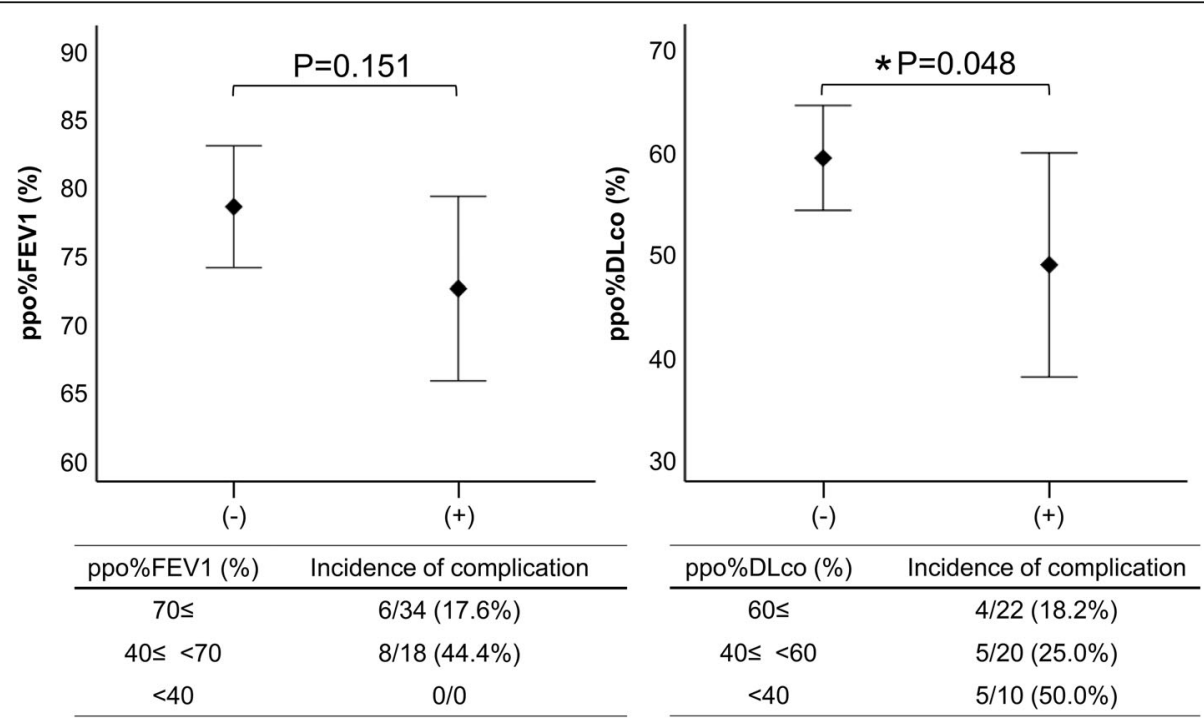

Fig. 5 Relationship between perioperative complications and postoperative predictive value. Differences between predicted postoperative \% forced expiratory volume in $1 \mathrm{~s}$ (ppo\%FEV1) and predicted postoperative \% diffusing capacity for carbon monoxide (ppo\%DLco) for the prediction of perioperative respiratory complications 
Table 3 Comparison between dynamic perfusion digital radiography and perfusion scintigraphy

\begin{tabular}{lll}
\hline & Dynamic perfusion digital radiography & Perfusion scintigraphy \\
\hline Cost & Low equipment cost + Work station & High equipment cost \\
Supplies & Unnecessary & Radionuclide: $^{99 \mathrm{~m}} \mathrm{Tc}-\mathrm{MAA},{ }^{99 \mathrm{~m}} \mathrm{Tc}-\mathrm{GAS}$ \\
Shooting time & $7 \mathrm{~s} \sim$ & $20 \mathrm{~min} \sim$ \\
Radiation dose & $0.09 \mathrm{mSv}$ & $0.8 \mathrm{mSv}$ \\
Procedure & Not established & Established \\
Information of result & Same as X-ray image & One-sided lung and lobe \\
& Functional information can be obtained & Established \\
\hline
\end{tabular}

and lung function tests, and the evaluation of intrathoracic tracheal narrowing in patients with COPD [25-28]. DCR can provide information not only in the supine position but also in a more physiological respiratory state of breathing, depending on the patient's position (standing or sitting). Ventilation imaging can observe not only atelectasis but also the ventilation distribution in the lung field, along with the observation of uneven distribution reflecting the air trapping associated with diffuse peripheral airway lesions, such as asthma. In addition, it is possible to observe not only the lung field but also the movement of the skeleton and diaphragm; therefore, it is possible to evaluate the differences in the expansion of the lung parenchyma in the upper and lower lung fields, such as IPF. Moreover, DPDR allows blood stream kinetics assessment, such as blood flow change for each heartbeat, and ventilation/perfusion ratio mismatch determined from ventilation and perfusion imaging at the same time axis. We believe that a portable DPDR system for emergency medicine within disaster areas will be made available in the diagnosis of pulmonary thromboembolism in the near future. As a result, a lot of information from the respiratory and circulatory kinetics in normal imaging and ventilation/ blood flow imaging by image processing could be easily obtained, and it may be widely used for purposes from disease diagnosis to functional evaluation in the future. Thus, the study seems to provide considerable incentives for exploring the respiratory and cardiovascular physiologies [16].

The current study has some limitations. First, this was a single-center study that included a small sample size. Moreover, this study was limited to surgery cases and excluded those with poor lung function from surgery, which likely resulted in bias. Furthermore, preoperative PPS was not performed in all cases to minimize exposure. Instead, it was only performed in cases with ventilatory and/or diffusion impairment. Second, DPDR does not directly measure actual lung perfusion but provides relative functional information related to lung circulation. Thus, some differences between DPDR and PPS findings are not surprising as both have completely different imaging targets, imaging mechanisms, and imaging postures. For instance, DPDR relies on pulmonary arterial blood flow to capture pulsations; whereas, PPS captures the accumulation of ${ }^{99 \mathrm{~m}} \mathrm{Tc}$-MAA microemboli in peripheral capillaries. Thus, examining cases with different degrees of pulmonary function will be essential in the future to reveal more accurate correlations.

\section{Conclusions}

Our study demonstrates that DPDR, which is simple, inexpensive, and makes obtaining respiratory functional information easy, can be a suitable substitute for PPS to predict postoperative values and the risk for postoperative respiratory complications. Nonetheless, further multicenter studies with larger sample sizes are needed to confirm our findings. This study provides novel insights using a new imaging system and demonstrates the possibility for functional analysis using DPDR.

\section{Supplementary Information}

The online version contains supplementary material available at https://doi. org/10.1186/s12957-021-02158-w.

Additional file 1. Comparison between predicted and measured values on forced expiratory volume in $1 \mathrm{~s}$ (FEV1). Correlations between FEV1 values predicted from dynamic perfusion digital radiography (DPDR) and pulmonary perfusion scintigraphy (PPS) and those measured using spirometry at postoperative months $1(\mathrm{a}, \mathrm{c})$ and $3(\mathrm{~b}, \mathrm{~d})$

\section{Abbreviations}

DCR: Dynamic chest radiography; DPDR: Dynamic perfusion digital radiography; PPS: Pulmonary perfusion scintigraphy; BFR: Blood flow ratio; FEV1: Forced expiratory volume in $1 \mathrm{~s}$; DLco: Diffusing capacity for carbon monoxide

\section{Acknowledgements}

We would like to thank Enago (www.enago.jp) for the English language review.

Katsunori Miyata, a radiological technician in our institution, contributed substantially by assisting with DCR.

\section{Authors' contributions}

$\mathrm{JH}$ : Conceptualization, data curation, formal analysis, methodology, and supervision. MY: Investigation. KH: Investigation. TS: Investigation. KO: 
Resources. RK: Resources. YK: Resources. YO: Resources. Akinaga Sonoda: Project administration. The author(s) read and approved the final manuscript.

\section{Funding}

This work was supported by Konica Minolta, Inc.

\section{Availability of data and materials}

The datasets used and/or analyzed during the current study are available from the corresponding authors on reasonable request.

\section{Ethics approval and consent to participate}

All procedures performed in this study were conducted in accordance with the ethical standards of the ethics committee and national research committee and in accordance with the 1964 Helsinki Declaration and its later amendments. The Institutional Review Board of Shiga University of Medical Science approved this study (CRB 5180008; 10 October 2017). The study was also registered as a clinical trial (UMIN000029716).

\section{Consent for publication}

Written informed consent was obtained from each participant in this study.

\section{Competing interests}

$\mathrm{JH}$ received a research grant from Konica Minolta, Inc. The remaining authors (MY, KH, TS, KO, RK, YK, YO, and AK) have no potential conflicts of interest related with this article.

\section{Author details}

'Division of General Thoracic Surgery, Department of Surgery, Shiga University of Medical Science, Shiga, Japan. 'Department of Radiology, Shiga University of Medical Science, Shiga, Japan.

\section{Received: 21 September 2020 Accepted: 31 January 2021}

Published online: 09 February 2021

\section{References}

1. Bray F, Ferlay J, Soerjomataram I, Siegel RL, Torre LA, Jemal A. Global cancer statistics 2018: GLOBOCAN estimates of incidence and mortality worldwide for 36 cancers in 185 countries. CA Cancer J Clin. 2018;68:394-424.

2. Ferguson MK, Watson S, Johnson E, Vigneswaran WT. Predicted postoperative lung function is associated with all-cause long-term mortality after major lung resection for cancer. Eur J Cardiothorac Surg. 2014:45:660-4.

3. Sawabata N, Nagayasu T, Kadota Y, Goto T, Horio H, Mori T, et al. Risk assessment of lung resection for lung cancer according to pulmonary function: republication of systematic review and proposals by guideline committee of the Japanese Association for Chest Surgery 2014. Gen Thorac Cardiovasc Surg. 2015;63:14-21.

4. Olsen GN, Block AJ, Tobias JA. Prediction of post pneumonectomy pulmonary function using quantitative macroaggregate lung scanning. Chest. 1974:66:13-6.

5. Bolliger CT, Gückiel C, Engel H, Stohr S, Wyser CP, Schoetzau A, et al. Prediction of functional reserves after lung resection: comparison between quantitative computed tomography, scintigraphy, and anatomy. Respiration. 2002;69:482-9

6. Wernly JA, DeMeester TR, Kirchner PT, Myerowitz PD, Oxford DE, Golomb HM. Clinical value of quantitative ventilation-perfusion lung scans in the surgical management of bronchogenic carcinoma. J Thorac Cardiovasc Surg. 1980;80:535-43.

7. Zeiher BG, Gross TJ, Kern JA, Lanza LA, Peterson MW. Predicting postoperative pulmonary function in patients undergoing lung resection. Chest. 1995:108:68-72.

8. Katarzyna KK, Józef K, Łukasz P, Małgorzata B, Paweł C, Jacek K, et al. Perfusion lung scintigraphy for the prediction of postoperative residual pulmonary function in patients with lung cancer. Nucl Med Rev. 2015;18: 70-7.

9. Tommaso CM, Orazio S, Eugenio P, Davide M, Giovanni S. Usefulness of lung perfusion scintigraphy before lung cancer resection in patients with ventilatory obstruction. Ann Thorac Surg. 2006:82:1828-34.

10. Giordano A, Calcagni ML, Meduri G, Valente S, Galli G. Perfusion lung scintigraphy for the prediction of postlobectomy residual pulmonary function. Chest. 1997;111:1542-7.
11. Abdel-Dayem HM, Scott A, Macapinlac H, Larson S. Tracer imaging in lung cancer. Eur J Nucl Med. 1994:21:57-81.

12. Ohno Y, Koyama H, Nogami M, Takenaka D, Matsumoto S, Yoshimura M, et al. Postoperative lung function in lung cancer patients: comparative analysis of predictive capability of MRI, CT, and SPECT. Am J Roentgenol. 2007;189:400-8

13. Oswald NK, Halle-Smith J, Mehdi R, Nightingale P, Naidu B, Turner AM Predicting postoperative lung function following lung cancer resection: a systematic review and meta-analysis. EClinicalMedicine. 2019;15:7-13.

14. Brunelli A, Kim AW, Berger Kl, Addrizzo-Harris DJ. Physiologic evaluation of the patient with lung cancer being considered for resectional surgery: diagnosis and management of lung cancer, 3rd ed: American College of Chest Physicians evidence based clinical practice guidelines. Chest. 2014; 143:e166S-90S. https://doi.org/10.1378/chest.12-2395.

15. Tanaka R, Sanada S, Okazaki N, Kobayashi T, Fujimura M, Yasui M, et al. Evaluation of pulmonary function using breathing chest radiography with a dynamic flat panel detector: primary results in pulmonary diseases. Invest Radiol. 2006;41:735-45.

16. Yamada Y, Ueyama M, Abe T, Araki T, Abe T, Nishino M, et al. Time-resolved quantitative analysis of the diaphragms during tidal breathing in a standing position using dynamic chest radiography with a flat panel detector system ("dynamic x-ray phrenicography"): initial experience in 172 volunteers. Acad Radiol. 2017;24:393-400.

17. Tanaka R. Dynamic chest radiography: flat-panel detector (FPD) based functional X-ray imaging. Radiol Phys Technol. 2016;9:139-53.

18. Bryant AS, Rudemiller K, Cerfolio RJ. The 30- versus 90-day operative mortality after pulmonary resection. Ann Thorac Surg. 2010;89:1717-23.

19. Kim AW, Boffa DJ, Wang Z, Detterbeck FC. An analysis, systematic review, and meta-analysis of the perioperative mortality after neoadjuvant therapy and pneumonectomy for non-small cell lung cancer. J Thorac Cardiovasc Surg. 2012;143:55-63.

20. Ali MK, Mountain CF, Ewer MS, Johnston D, Haynie TP. Predicting loss of pulmonary function after pulmonary resection for bronchogenic carcinoma. Chest. 1980;77:337-42.

21. Gass GD, Oslen GN. Preoperative pulmonary function testing to predict postoperative morbidity and mortality. Chest. 1986;89:127-35.

22. Bland JM, Altman DG. Statistical methods for assessing agreement between two methods of clinical measurement. Lancet. 1986;1:307-10.

23. Pierce RJ, Copland JM, Sharpe K, Barter CE. Preoperative risk evaluation for lung cancer resection: predicted postoperative product as a predictor of surgical mortality. Am J Respir Crit Care Med. 1994;150:947-55.

24. Markos J, Mullan BP, Hillman DR, Musk AW, Antico VF, Lovegrove FT, et al. Preoperative assessment as a predictor of mortality and morbidity after lung resection. Am Rev Respir Dis. 1989;139:902-10.

25. Yamasaki Y, Abe K, Hosokawa K, Kamitani T. A novel pulmonary circulation imaging using dynamic digital radiography for chronic thromboembolic pulmonary hypertension. Eur Heart J. 2020;41:2506.

26. Tamura M, Matsumoto I, Saito D, Yoshida S, Takata M, Takemura H. Case report: uniportal video-assisted thoracoscopic resection of a solitary fibrous tumor preoperatively predicted visceral pleura origin using dynamic chest radiography. J Cardiothoracic Surg. 2020;15:166.

27. Ohkura N, Kasahara K, Watanabe S, Hara J, Abo M, Sone T, et al. Dynamicventilatory digital radiography in air flow limitation: a change in lung area reflects air trapping. Respiration. 2020:99:382-8.

28. Watase S, Sonoda A, Matsutani N, Muraoka S, Hanaoka J, Nittaa N, et al. Evaluation of intrathoracic tracheal narrowing in patients with obstructive ventilatory impairment using dynamic chest radiography: a preliminary study. Eur J Radiol. 2020;129:109141.

\section{Publisher's Note}

Springer Nature remains neutral with regard to jurisdictional claims in published maps and institutional affiliations. 